\title{
An Electrostatic Charge Partitioning Model for the Dissociation of Protein Complexes in the Gas Phase
}

\author{
Stephen V. Sciuto, Jiangjiang Liu, Lars Konermann \\ Department of Chemistry, The University of Western Ontario, London, Ontario, N6A 5B7, Canada
}

\begin{abstract}
Electrosprayed multi-protein complexes can be dissociated by collisional activation in the gas phase. Typically, these processes follow a mechanism whereby a single subunit gets ejected with a disproportionately high amount of charge relative to its mass. This asymmetric behavior suggests that the departing subunit undergoes some degree of unfolding prior to being separated from the residual complex. These structural changes occur concomitantly with charge (proton) transfer towards the subunit that is being unraveled. Charge accumulation takes place up to the point where the subunit loses physical contact with the residual complex. This work develops a simple electrostatic model for studying the relationship between conformational changes and charge enrichment during collisional activation. Folded subunits are described as spheres that carry continuum surface charge. The unfolded chain is envisioned as random coil bead string. Simulations are guided by the principle that the system will adopt the charge configuration with the lowest potential energy for any backbone conformation. A finite-difference gradient algorithm is used to determine the charge on each subunit throughout the dissociation process. Both dimeric and tetrameric protein complexes are investigated. The model reproduces the occurrence of asymmetric charge partitioning for dissociation events that are preceded by subunit unfolding. Quantitative comparisons of experimental MS/MS data with model predictions yield estimates of the structural changes that occur during collisional activation. Our findings suggest that subunit separation can occur over a wide range of scission point structures that correspond to different degrees of unfolding.
\end{abstract}

Key words: Protein complex, Noncovalent interaction, Electrospray mass spectrometry, Hemoglobin, Asymmetric charge partitioning

\section{Introduction}

- lectrospray ionization mass spectrometry (ESI-MS) has 1 become a well established tool for the characterization of noncovalent protein-protein and protein-ligand complexes [1-7]. The gentle nature of ESI [8] and nanoESI [9] allows the transfer of intact biological assemblies into the gas phase as multiply protonated species (assuming that measurements are conducted in positive ion mode). The binding stoichiometries of these complexes can be determined on the basis of mass measurements. Also, affinity

Correspondence to: Lars Konermann; e-mail: konerman@uwo.ca estimates can be obtained based on signal intensity ratios of the free and bound species [10-14]. Key to the success of these studies is the use of experimental conditions that facilitate gentle ion sampling [15, 16], and that minimize inadvertent dissociation [17-19] or nonspecific clustering $[20,21]$.

Mounting evidence suggests that protein complexes in the gas phase can retain much of their solution structure [22]. This correlation greatly expands the scope of gas phase structural investigations such as ion mobility measurements [22-24], gas phase hydrogen exchange [25], and optical spectroscopy [26]. In addition, a host of ion activation methods are available for the dissociation of protein assemblies [27-32]. 
Collision-induced dissociation (CID) represents the most widely used approach for the fragmentation of biomolecular ions in the gas phase. CID may be conducted on different types of mass spectrometers and at various physical locations along the ion path, e.g., in rf-only multipoles, traveling-wave ion guides, ion cyclotron resonance cells, and in the ion sampling interface [30, 33]. CID involves relatively slow (microsecond) heating of analyte ions, mediated by multiple collisions with neutral gas molecules [34]. When applied to protein-protein complexes, CID conditions are typically chosen such that noncovalent subunit interactions are disrupted while covalent bonds remain intact $[29,35]$.

An intriguing phenomenon during CID of multi-protein assemblies is the prevalence of fragmentation pathways whereby a single subunit is ejected with a disproportionately high amount of charge relative to its mass. The first example of this "asymmetric charge partitioning" was reported by Schwartz et al. [7] who observed that CID of streptavidin $[4 \mathrm{M}+14 \mathrm{H}]^{14+}$ ions yields $[\mathrm{M}+7 \mathrm{H}]^{7+}$ and $[3 \mathrm{M}+7 \mathrm{H}]^{7+}$ ions. In this example, the monomeric product ion carries half of the precursor charge, but only a quarter of the mass. Similar effects have been observed for numerous other protein complexes, ranging in size from dimers to multi-subunit assemblies [3, 33, 36-41], as well as for DNA duplexes [42, 43]. The extent of asymmetry is somewhat dependent on the experimental conditions [33], but only very few systems exhibit completely symmetric CID behavior [36]. Asymmetric charge partitioning also occurs during blackbody infrared dissociation of protein-protein assemblies [31, 44].

A conceptual framework has evolved to rationalize the occurrence of asymmetric charge partitioning $[3,30,31,33$, $37,45,46]$. This framework is based on the view that electrosprayed multi-protein assemblies initially retain a compact conformation, where excess protons are distributed more or less uniformly on the surface of the complex. Collisional activation induces gradual unfolding of a single subunit, accompanied by electrostatically driven proton transfer to this subunit. This transfer of charge comes to an end when the partially unfolded subunit has passed the "scission point," i.e., when it has separated from the residual complex. The charges on the complementary CID product ions reflect the extent of proton transfer that has occurred up to the scission point.

The subunit unfolding framework outlined above is supported by several lines of evidence. (1) Proton mobility within collisionally activated polypeptide ions [47, 48] as well as charge transfer between protein subunits [31] are well documented. (2) Intramolecular crosslinks diminish the extent of charge asymmetry, in line with the expectation that subunit unfolding will be impeded under these conditions $[33,37]$. (3) Because unfolding is a time-dependent process, symmetric partitioning is favored when employing surfaceinduced dissociation (a picosecond process $[49,50]$ ) instead of CID, which proceeds on a microsecond time scale [30]. (4) Ion mobility measurements on collisionally activated multi-protein complexes confirm the presence of semiunfolded intermediate structures [35, 51, 52]. (5) Electrostatic models that allow for subunit unfolding yield data that are in qualitative agreement with experimental observations [44]. In contrast, models based on static subunit structures underestimate the extent of asymmetric charge partitioning $[45,53-55]$.

Although the general validity of the subunit unfolding framework is undisputed, several aspects related to asymmetric charge partitioning remain incompletely understood. For example, some theoretical studies predict that chargesymmetric behavior should generally be favored on the basis of kinetic arguments [46, 56]. Another aspect that has received little attention is the fact that CID product ions typically display a wide distribution of charge states, despite the use of precursor ions with a precisely defined number of excess protons. The last feature is particularly interesting, as it suggests the existence of competing fragmentation pathways. Some previous computational investigations treated charge migration by using manual intervention to move protons among amino acid side chains [44, 46, 56]. Strategies of that type can make it difficult to adequately account for conformationally driven charge migration.

The goal of this study is to describe the CID process of noncovalent protein-protein complexes using a simple electrostatic model. We aim to account for product ion abundance distributions in a quantitative way, thereby providing insights into the protein structural changes occurring during collisional activation. This work incorporates ideas put forward in previous studies, particularly Klassen's protein structure model [44], Thachuk's Coulomb repulsion model $[46,56]$, the two-sphere model of Ryce and Wyman [53], and a droplet fission model developed by our group [57].

\section{Theoretical Framework}

The model developed here is applicable to protein complexes with different geometries and subunit compositions. For reasons of simplicity, we will first discuss a homodimer composed of two subunits, A and B, that both have the same initial radius $r_{1}$. Prior to collisional activation, both subunits are described as spheres, consistent with the globular fold of many protomers (Figure 1, top). During collisional activation, subunit $A$ undergoes structural changes, whereas the shape of subunit B remains unaltered.

Gradual subunit unfolding during collisional activation is described by having a string of small spherical beads emerge from subunit A. Each of the additional beads represents one residue, and the unraveling process occurs in a stepwise sequential fashion. A numbering scheme is introduced to refer to each entity of the system. Subunit B is designated as 1 , and the subunit $\mathrm{A}$ sphere is designated as 2 . Subsequent amino acid beads are numbered 3, 4, 5, etc. (Figure 1). All amino acid beads share the same radius $r_{a a}$. The amino acid string is described as a self-avoiding random coil in three 


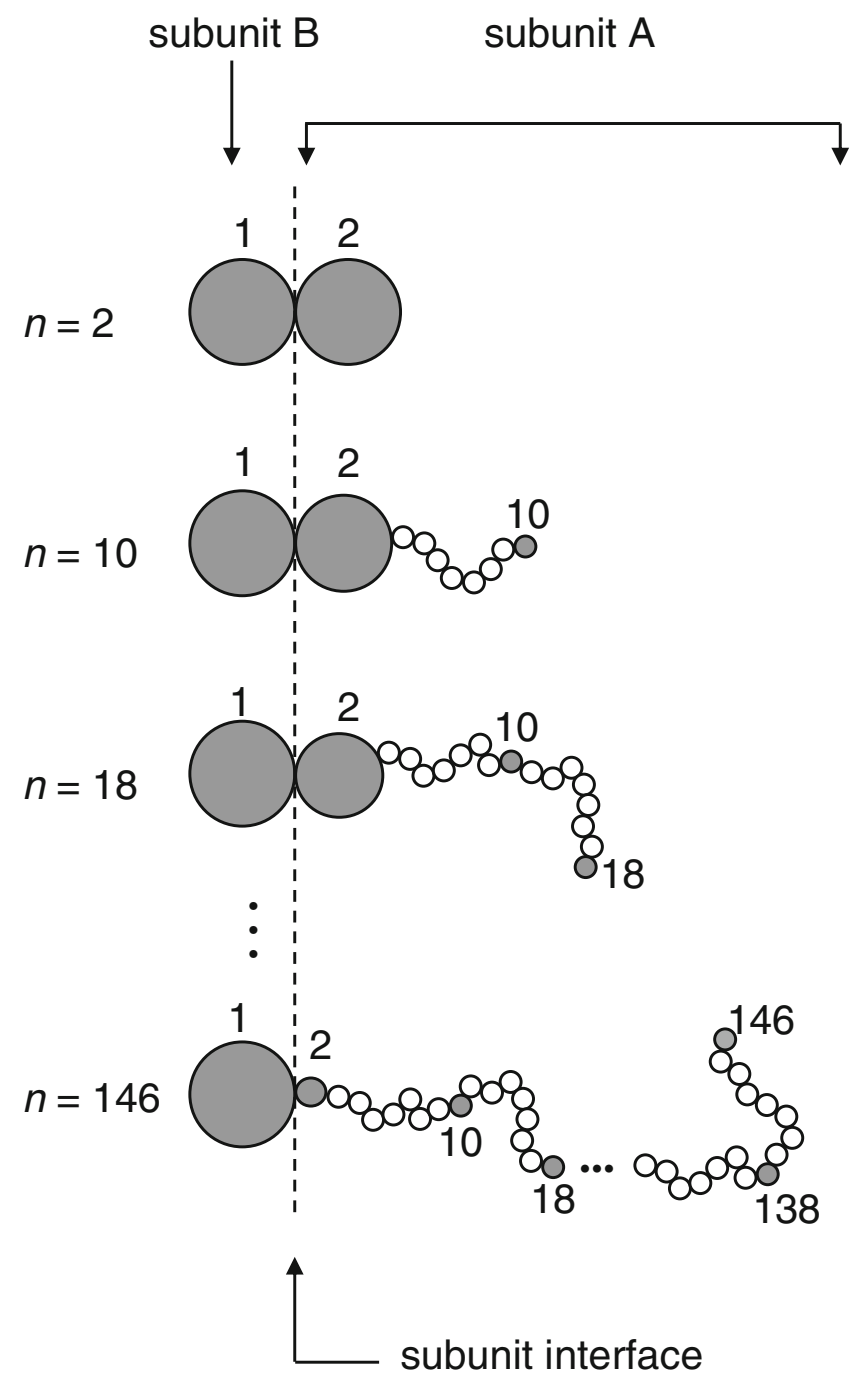

Figure 1. Schematic cartoon, depicting structural changes during collisional activation of a dimeric protein complex in the gas phase. Unfolding of subunit A occurs in a sequential manner. The growing amino acid chain adopts a random coil structure. The total number of spherical entities is denoted as $n$. Sites that can carry charge are highlighted in gray. Subunit separation can occur at any point during the process. For presentation purposes a large chain segment has been omitted in the bottom panel

dimensions. The degree to which subunit $\mathrm{A}$ has undergone unraveling is denoted by the parameter $n$ that corresponds to the total number of entities in the system. For example, $n=2$ represents the intact complex prior to collisional activation. Similarly, $n=10$ represents the case where eight unraveled amino acid beads are present, in addition to the large spheres 1 and 2. It is assumed that the fully unfolded subunit A consists of 151 residues. This number was chosen to facilitate subsequent comparisons with experimental data. The unraveling process is subject to conservation of volume. This implies that the radius of sphere 2 decreases with each amino acid that is added to the unfolded chain. The amino acid bead radius is given by the relationship $r_{1}{ }^{3}=151 r_{a a}{ }^{3}$.
Of the 20 amino acid side chains, those of Arg, His, and Lys, as well as the amino terminus represent the main protonation sites in gas phase proteins $[44,56,58]$. The average abundances of Arg, His, and Lys are 5.2\%, 2.1\%, and $6.1 \%$, respectively, corresponding to a total of $13.4 \%$ [59]. In order to closely match this percentage, every eighth residue $(10,18,26, \ldots)$ was designated as a possible charge attachment site, in addition to spheres 1 and 2 (gray in Figure 1).

The dissociation of multiply charged protein complexes after collisional activation shares conceptual analogies with the breakup of charged solvent droplets [45, 53, 57, 60-64]. Guided by those earlier investigations, excess proton charge is modeled using a continuum approximation. Specifically, the total charge of the system is assumed to be distributed over the surface of the electrifiable entities $(1,2,10,18,26$, ...) [65]. The electrostatic energy $E_{n}$ of the system is given by [57]

$$
E_{n}=\frac{1}{8 \pi \varepsilon_{0}}\left(\sum_{i=1}^{n} \frac{q_{i}{ }^{2}}{r_{i}}\right)+\frac{1}{4 \pi \varepsilon_{0}} \sum_{i=1}^{n-1} \sum_{j=i+1}^{n} \frac{q_{i} q_{j}}{r_{i j}}
$$

where $q_{i}$ is the charge on entity $i, r_{i}$ is the corresponding radius, and $\varepsilon_{0}$ is the vacuum permittivity. The variable $r_{i j}$ refers to the midpoint distance between $i$ and $j$. The first term in Eq. 1 reflects the electrostatic energy associated with accumulating a charge $q_{i}$ on all the spherical entities $i$, whereas the double sum accounts for repulsive interactions among the entities. As noted earlier, only a subset of entities are designated as chargeable sites $\left(q_{i} \geq 0\right)$, whereas $q_{i}=0$ for all others.

A central feature of the model is that for any unfolding stage $n$ the system adopts the charge configuration $q_{1}, \ldots$, $q_{n}$ corresponding to the global minimum of $E_{n}[53,57]$. This behavior reflects the fact that proton transfer occurs on a time scale that is much faster than subunit unfolding $[47,48,56]$. The charge configuration depends on the degree of subunit unraveling, as well as on the chain conformation. The latter affects the values of $r_{i j}$ in Eq. 1 . A comprehensive characterization of the system properties must therefore consider various different conformations for each value of $n$.

The most important observable predicted by the model is the amount charge that has accumulated on subunit A $\left(q_{A}\right)$ once the system has reached the scission point. Up to this stage, subunit A (entities 2, ..,n) and subunit B (entity 1) form a contiguous chain where charge can exchange freely between the subunits. Once CID has pushed the system past the scission point there will be an insulating gap at the subunit interface (Figure 1) such that $q_{A}$ and $q_{B}$ can undergo no further change [44]. Conformational transitions that occur after the scission point are irrelevant for charge partitioning among the subunits. The total amount of excess charge within the system is referred to as $q_{t o t}$. For a system that reaches the scission 
point at chain length $n$ the charge on complementary product ions is given by

$$
\begin{gathered}
q_{A}=\sum_{i=2}^{n} q_{i} \\
q_{B}=q_{t o t}-q_{A}
\end{gathered}
$$

Ultimately, the success of the model hinges on comparisons of predicted $q_{A}$ values with experimentally determined charge states after CID. Asymmetric charge partitioning corresponds to $q_{A}>q_{B}$, whereas the symmetric case is characterized by $q_{A} \approx q_{B}$.

An extension of the described dimer model to larger systems is straightforward. This work will also consider tetrameric complexes with equally sized spherical subunits A, B, C, D that initially adopt a tetrahedral arrangement. This layout mimics the quaternary structure displayed by many protein systems [66]. During collisional activation, subunit $\mathrm{A}$ undergoes unraveling in a fashion analogous to that described above. Subunits B, C, and D remain intact, and their relative positions do not change.

\section{Simulations}

Computer calculations were performed using FORTRAN code developed in-house. For dimer simulations two equally sized spheres were initially placed with their midpoints spaced by $2 r_{I}$ along the $x$-axis of a Cartesian coordinate system. Conservation of volume dictates that extrusion of the amino acid string during collisional activation is accompanied by gradual shrinkage of sphere 2 . The midpoint position of that sphere was adjusted throughout the simulation to ensure that spheres 1 and 2 remained in physical contact at all times. The midpoints of unfolded amino acid beads were placed in three dimensions with a step size of $2.5 r_{a a}$, and with spherical polar coordinate angles $0 \leq \theta<2 \pi$ and $0 \leq \phi<\pi$ that were picked using a pseudo-random number generator. The finite-difference gradient routine UMINF (IMSL; Visual Numerics, Houston, TX, USA) was used for determining the charge configuration $q_{1}$, $\ldots, q_{n}$ corresponding to the global minimum of $E_{n}$ (Eq. 1). 50 different random chain conformations were tested for any given value of $n$. Each of these 50 conformations represents a possible scission point structure. Extension of the model to a tetrameric protein complex was achieved as outlined above.

\section{Experimental}

Bovine $\beta$-lactoglobulin (BLG, pdb code 1BEB) was purchased from Sigma (St. Louis, MO, USA). Bovine hemoglobin ( $\mathrm{Hb}$, pdb code 2QSS) was isolated from fresh cow blood in its oxygenated $\left(\mathrm{Fe}^{2+}\right.$, ferro) form following established procedures [67]. CID of ferro-heme occurs primarily by neutral loss [68], such that no charge corrections had to be performed when comparing heme-free and heme-bound fragment ions. All mass spectra were recorded under gentle ESI condition using a quadrupoletime-of-flight instrument (Q-TOF Ultima; Waters, Milford, MA), employing instrument settings and solution conditions described previously [13]. The ESI source was operated in positive ion mode with a capillary voltage of $3 \mathrm{kV}$. Protein solutions containing $50 \mu \mathrm{M} \mathrm{Hb}$ (as tetramer) or BLG (as dimer) and $150 \mathrm{mM}$ ammonium acetate at $\mathrm{pH} 6.8$ were infused by a syringe pump (Harvard 22; Boston, MA, USA) at $5 \mu \mathrm{L} \mathrm{min}$. CID was conducted in the hexapole collision cell of the Q-TOF instrument with $\mathrm{Ar}$ as collision gas. Collision energies were between 30 and $60 \mathrm{~V}$, ensuring that residual parent ion signals in the tandem mass spectra were comparable to those of the fragment ions.

\section{Results and Discussion}

\section{Dimer Simulations}

The model outlined above was used to study the relationship between subunit unfolding and charge partitioning during collisional activation of a protein complex. We will first discuss results obtained for a dimer. The histograms in Figure $2 \mathrm{a}-\mathrm{f}$ display the charge percentage on subunit $\mathrm{A}$ for different values of $n$, representing the degree of unfolding at the scission point. In the absence of unfolding subunit A holds $50 \%$ of the charge after subunit separation (Figure 2a). With increasing $n$, additional charge migrates towards subunit $\mathrm{A}$ as the system maintains the state with lowest electrostatic energy. In cases where subunit $\mathrm{A}$ is almost completely unraveled it carries $\sim 65 \%$ of the overall charge ( $n=146$, Figure 2f). This last scenario corresponds to a high degree of asymmetry, with a $\sim 2: 1$ charge ratio of the product ions $\mathrm{A}$ and $\mathrm{B}$, and a 1:1 mass ratio. Under these conditions the $65 \%$ charge is distributed relatively uniformly among the electrifiable amino acid beads of subunit A (data not shown).

Each histogram in Figure 2a-f represents simulation data for fifty different chain conformations. The resulting distributions are quite narrow, with fwhm values on the order of $5 \%$. In other words, different chain conformations result in similar charge partitioning as long as the degree of subunit unfolding $(n)$ remains the same.

The dimer model was also used to calculate discretized charge state distributions of subunit A, for a precursor charge of $13+$ (Figure 2g-1). These data were obtained using a procedure analogous to that described above, but with the restriction that charge had to appear in integer values. The purpose of this alternative representation is to facilitate subsequent comparisons with experimental data. The model predicts that CID of a 13+ dimer precursor without unfolding produces monomeric $7+$ and $6+$ product ions in equal abundance (Figure $2 \mathrm{~g}$ ). In contrast, charge states $9+$ and $8+$ are expected for subunit $\mathrm{A}$ in the case of extensive unfolding (Figure 21). 


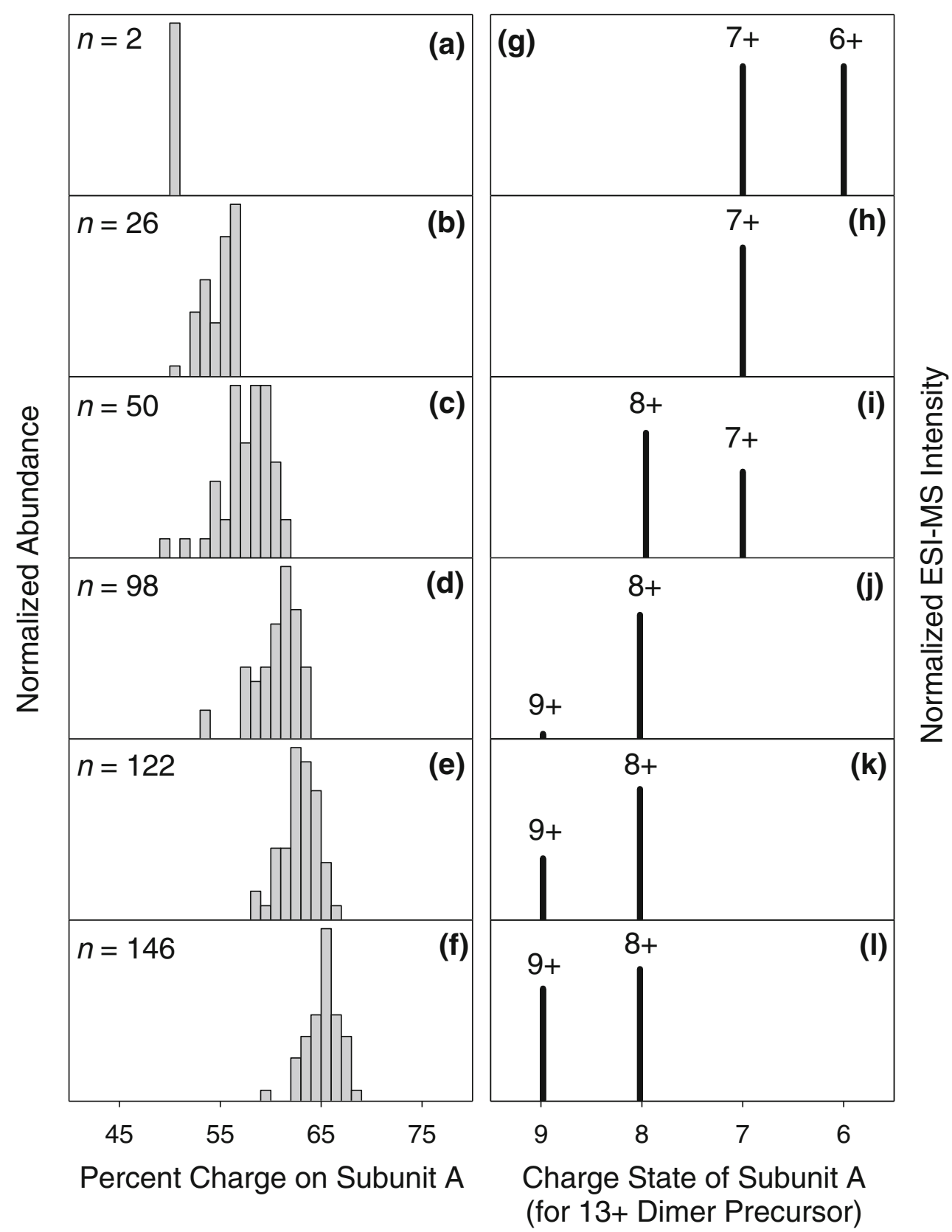

Figure 2. Simulated charge distributions for subunit A of a dimeric protein that undergoes collisionally activated unfolding prior to reaching the scission point. For $n=2$ the subunit is folded $(\mathrm{a}, \mathrm{g})$; for $n=146$ the subunit is extensively unfolded (f, I). Panels (a)-(f) refer to charge percentages, reflecting the continuum nature of the model. Panels (g)-(l) are for integer charge values, assuming a $13+$ precursor ion. Panels $\mathbf{( g )}-\mathbf{( I )}$ are plotted on a reversed $x$-axis to facilitate comparisons with experimental data. Subunit $B$ remains folded in all cases

\section{Tetramer Simulations}

The modeling procedure was extended to explore the CID behavior of a noncovalent tetramer that initially consists of four identically sized subunits (Figure 3). Subunit A undergoes unraveling during collisional activation, whereas $\mathrm{B}, \mathrm{C}$, and $\mathrm{D}$ remain intact. Note that the value of $n$ has to be shifted by two in order to account for the two additional subunits. Hence, $n=4$ refers to the case where all four subunits remain folded. Not surprisingly, subunit A is predicted to carry $25 \%$ of the total charge when it is ejected at this early stage (Figure 3a). Subunit unfolding during collisional activation leads to charge accumulation, up to $\sim 55 \%$ for $n=148$ (Figure 3f). While this value is smaller than the $\sim 65 \%$ predicted for the dimer (Figure 2f), one has to consider that the unfolded chain competes for charge with three other subunits in the tetramer. Hence, the ejection of subunit A with more than half of the total charge represents a dramatic case of asymmetric charge partitioning. 


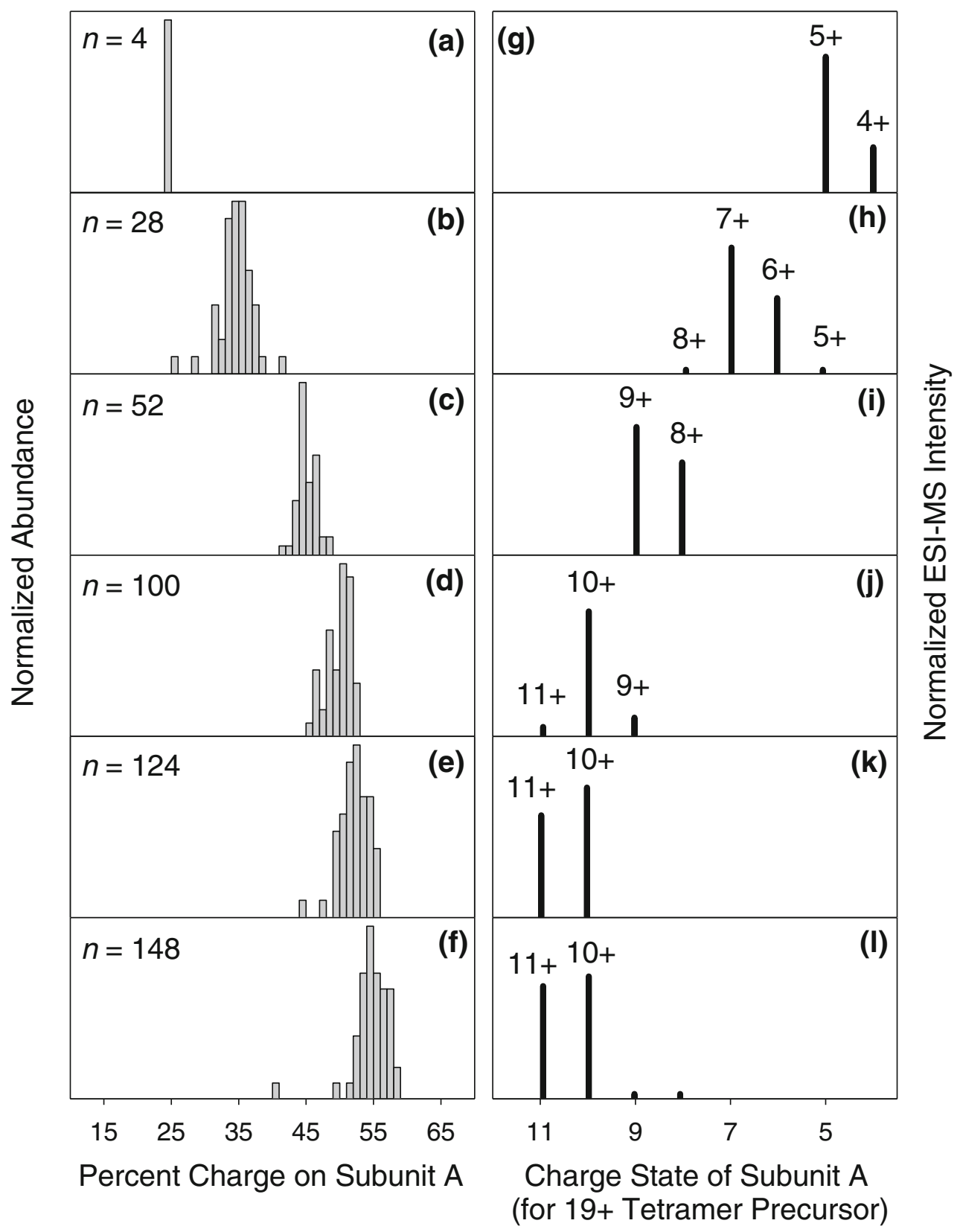

Figure 3. Simulated charge distributions for subunit A of a tetrameric protein that undergoes collisionally activated unfolding prior to reaching the scission point. Notation and layout are analogous to Figure 2. Panels (g) -(I) are for a 19+ tetrameric precursor ion

Discretized charge state distributions for CID of the tetramer were calculated for a precursor ion charge state of 19+ (Figure 3g-1). Under these conditions the symmetric case corresponds to a charge state distribution that is dominated by $5+$, with a 3 -fold less intense peak for $4+$ (Figure $3 \mathrm{~g}$ ). Extensive unfolding of subunit A yields charge states $11+$ and $10+$ with similar intensities (Figure 3f).

\section{Experimental CID Data}

The protein systems investigated in this work, $\beta$-lactoglobulin (BLG) $[13,21,69]$ and hemoglobin $(\mathrm{Hb})[67,70-72]$, have previously been characterized by ESI-MS. The number of amino acids per subunit is comparable for the two systems. BLG forms homodimers [73] with 162 residues $(18,281 \mathrm{Da})$ per chain. The $\alpha$ subunit of $\mathrm{Hb}$ consists of 141 residues $(15,053 \mathrm{Da})$, whereas the $\beta$ subunit has 145 amino acids $(15,954 \mathrm{Da})$. Under native conditions, every $\mathrm{Hb}$ subunit is bound to a heme group (h). ESI mass spectra of native $\mathrm{Hb}$ are dominated by tetramer ions with the composition $\alpha_{2} \beta_{2} \mathrm{~h}_{4}[13,67,72]$. In addition, the spectra also reveal the presence of dimers $\left(\alpha \beta h_{2}\right)[13,67,72]$.

CID of BLG $13+$ dimers results in dominant signals for monomeric $8+$ and $7+$ ions, as well as the complementary $5+$ 
and $6+$ species (Figure $4 \mathrm{a}$ ). MS/MS of $\left[\alpha \beta \mathrm{h}_{2}+13 \mathrm{H}\right]^{13+}$ yields dominant heme-free $\alpha$ and $\beta$ product ions in charge states $9+$ to $7+$ (Figure $4 b)$. CID of $\left[\alpha_{2} \beta_{2} h_{4}+19 \mathrm{H}\right]^{19+}$ results in a spectrum that is dominated by heme-free monomers in charge states $11+$ to $8+$ (Figure $4 \mathrm{c}$ ).

The fragmentation patterns of Figure 4 are consistent with earlier observations [40,74]. In the case of $\mathrm{Hb}$ (Figure 4b, c), product ions in the high $\mathrm{m} / \mathrm{z}$ range show exceedingly low signal intensities. This effect is common in TOF-MS-based CID studies on protein complexes. Its origin has been traced to focusing issues inside flight tube, as well as discrim-

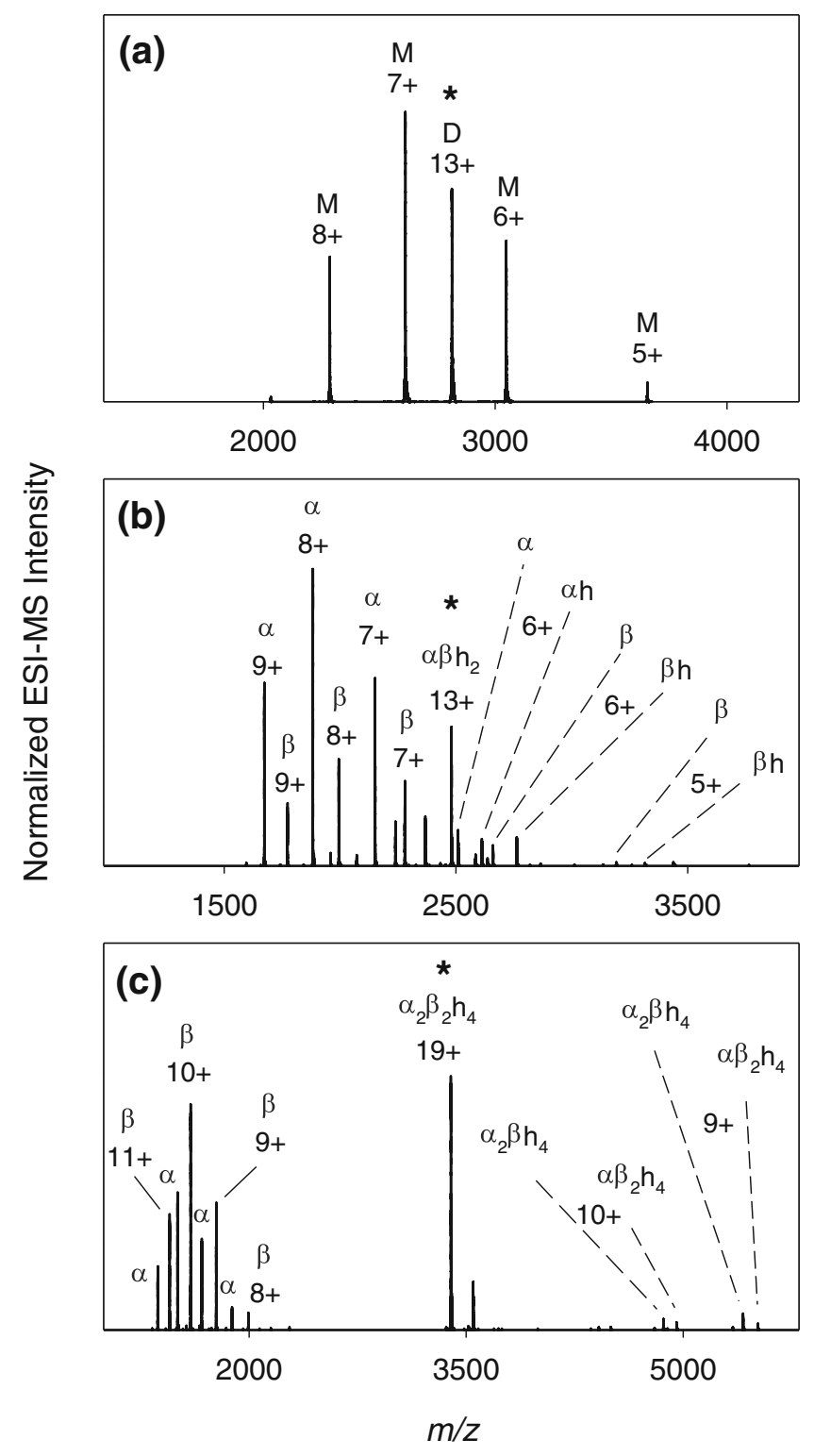

Figure 4. Mass spectra recorded after CID of three protein complexes. Residual precursor ion signals are denoted with asterisks. (a) MS/MS of $[\mathrm{BLG} \text { dimer }+13 \mathrm{H}]^{13+}$. Dimer and monomer are denoted as $D$ and $M$, respectively. (b) MS/MS of dimeric $\mathrm{Hb}$ ions, $\left[\alpha \beta \mathrm{h}_{2}+13 \mathrm{H}\right]^{13+}$. Subunits are denoted as $\alpha$ and $\beta ; \mathrm{h}$ represents heme. (c) MS/MS of $\mathrm{Hb}$ tetramers $\left[\alpha_{2} \beta_{2} h_{4}+19 H\right]^{19+}$ ination effects at the detector $[49,54]$. For the present work, this effect is of little relevance, as our attention is on fragment ions that appear in the $\mathrm{m} / \mathrm{z}$ range below the precursor ion.

Figure 4 indicates that the degree of charge asymmetry is different for the three complexes considered here. BLG 13+ dimers show the most symmetric behavior, with 7+ monomers as the dominant CID product. The low level of asymmetry is consistent with the presence of two disulfide linkages in each subunit, which limit the extent of unraveling during collisional activation $[33,37]$. Charge asymmetry is more pronounced for the $\mathrm{Hb} 13+$ dimer, which yields monomeric CID fragments in charge states up to 9+. The most dramatic example of charge asymmetry is displayed by $\mathrm{Hb}$ tetramers where dissociation of the 19+ precursor produces monomers up to $11+\mathrm{Hb}$ subunits do not possess any disulfide bridges.

The major high charge state CID products generated from $\mathrm{Hb}$ dimers correspond to $\alpha$, whereas $\beta$ is dominant during fragmentation of the tetramer (Figure $4 b, c$ ). The origin of this behavior is not clear, but it is likely related to the specific subunit interactions in the two types of complexes. The intensity progressions of $\alpha$ and $\beta$ within each of the two tandem mass spectra are very similar. Both $\mathrm{Hb}$ subunits exhibit a high sequence homology and they adopt similar native state structures [66]. To facilitate the following modeling considerations, we will not distinguish between the two $\mathrm{Hb}$ subunit types.

\section{Comparison of Model and Experiments}

The electrostatic model developed in this work (Figures 1-3) confirms that charge partitioning during CID will be strongly asymmetric in cases where the ejected subunit has undergone unfolding prior to reaching the scission point. This prediction is consistent with experimental observations. For example, Schwartz et al. [45] reported that collisional activation of a tetramer leads to ejection of monomers carrying (on average) $50 \%$ of the total charge. This behavior is readily accounted for in the model when assuming that the departing subunit is ejected at the $n \approx 100$ stage (Figure $3 \mathrm{~d}$ ). In order to make more detailed comparisons between theory and experiment, a few additional considerations are required.

The model predicts that the fragment ion charge state distributions for any scission point $n$ will be quite narrow, with major peaks that cover no more than two protonation states (Figures $2 \mathrm{~g}-1,3 \mathrm{~g}-1$ ). In contrast, experimental spectra often reveal a much higher degree of charge heterogeneity $[3,33,36-41]$. This disparity implies that subunit separation does not always occur at the same value of $n$ during collisional activation of a precursor ion population. Instead, complexes must undergo subunit separation over a range of scission point structures that represent different degrees of unfolding.

The realization that CID of protein complexes involves competing fragmentation pathways (corresponding to differ- 
ent scission points) provides a strategy for the quantitative analysis of experimental MS/MS data. We will initially discuss the fragmentation of a dimer in the $13+$ charge state. Each of the simulated monomer charge state distributions in Figure $2 \mathrm{~g}-\mathrm{l}$ is interpreted as a basis function $\Phi_{n}$. As before, $n$ refers to the degree of subunit unraveling at the scission point. The contributions of different fragmentation channels are accounted for by generating fitted charge state distributions $f$ as linear combination

$$
f=\sum_{n=1}^{m} c_{n} \Phi_{n}
$$

where the magnitude of each coefficient $c_{n}$ represents the weighting of scission point structure $n . m$ is the number of relevant scission point structures. A slight complication is the fact that the $\Phi_{n}$ do not represent an orthogonal basis set. Thus, different $c_{n}$ combinations may result in the same $f$. For this reason, the following analyses strive to describe experimental data using the lowest possible number of $c_{n}$ coefficients. This strategy provides qualitative insights into the range of relevant types of scission point structures. To further simplify the data interpretation, we will not consider each possible stage of subunit unfolding, but only $n=2,10$, $18, \ldots$ as indicated in Figure 1.

Figure $5 \mathrm{a}$ reveals that the fragment ion charge state distribution obtained after collisional activation of BLG 13+ dimers is well described by the linear combination

$$
f(\text { BLG dimer })=0.3 \Phi_{34}+0.7 \Phi_{42}
$$

In contrast, data obtained for the $\mathrm{Hb} 13+$ dimer require a completely different set of basis functions, providing an adequate description for $\alpha$ as well as $\beta$ product ions (Figure 5b).

$$
f(\mathrm{Hb} \text { dimer })=0.34 \Phi_{26}+0.27 \Phi_{98}+0.42 \Phi_{138}
$$

The basis functions $\Phi_{n}$ used in Eqs. 4 and 5 reflect major differences in the degree of subunit unfolding between BLG and $\mathrm{Hb}$ dimers. In the case of BLG, the departing subunit remains relatively compact at the scission point, as there is no evidence for the involvement of $n$ values higher than $\sim 42$. Dissociation of $\mathrm{Hb}$ dimers involves a much wider range of scission point structures, reaching from $n \approx 26$ all the way to chains that are almost completely unfolded $(n \approx 138)$. The tendency of BLG to pursue fragmentation pathways with lower degrees of subunit unraveling is consistent with the presence of intra-subunit disulfide bridges, as noted above.

The linear combination strategy introduced above can also be extended to tetrameric complexes. In this case the simulated distributions of Figure $3 \mathrm{~g}-1$ (for a $19+$ precursor ion) are used as basis functions $\Phi_{n}$. Application of this strategy to the $\mathrm{Hb}$ tetramer CID data (Figure 5c) yields the following parameters:

$$
f(\text { Hb tetramer })=0.22 \Phi_{60}+0.32 \Phi_{92}+0.42 \Phi_{132}
$$

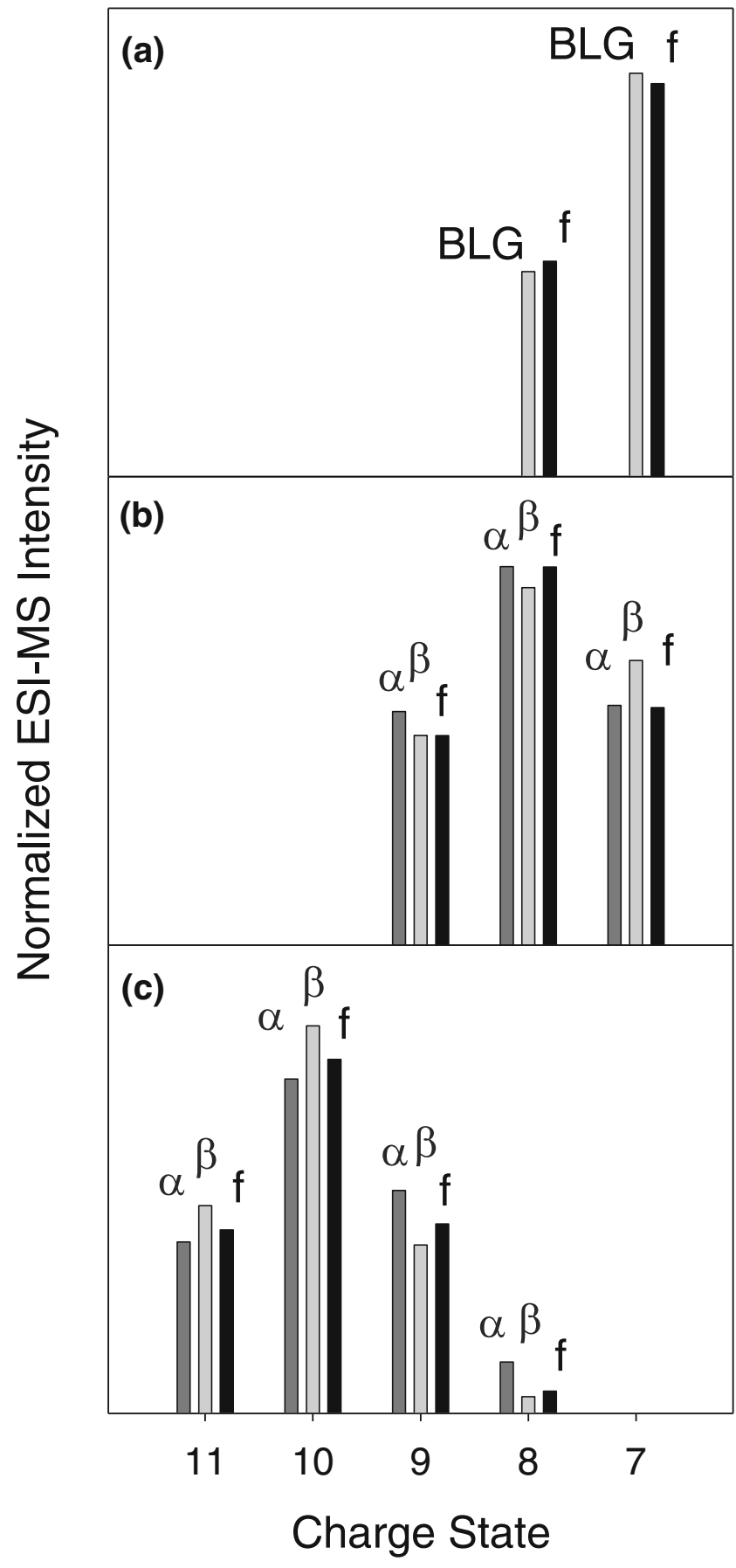

Figure 5. Experimental and fitted charge state distributions for monomeric fragments generated by CID of (a) [BLG dimer $+13 \mathrm{H}]^{13+}$, (b) $\mathrm{Hb}$ dimer $\left[\mathrm{a} \beta \mathrm{Sh}_{2}+13 \mathrm{H}\right]^{13+}$, and (c) $\mathrm{Hb}$ tetramer $\left[\alpha_{2} \beta_{2} h_{4}+19 H\right]^{19+}$. All data were normalized to provide the same integrated ESI-MS intensity. Experimental intensity progressions were obtained from the spectra of Figure 4. Fitted peak distributions (black, marked as " $f$ " in each panel) were generated as linear combinations of modeled basis spectra (eq 3)

This analysis reveals that $\mathrm{CID}$ of $\mathrm{Hb}$ tetramers is dominated by fragmentation pathways that involve extensive unfolding of the departing subunit, similar to the $\mathrm{Hb}$ dimers 
discussed above. For the dimer, however, some of the complexes undergo subunit separation quite early during collisional activation $\left(\Phi_{26}\right.$, Eq. 5). In contrast, subunit separation for the tetramer does not occur until the $n \approx 60$ stage has been reached. This difference likely reflects the extent to which the unraveling subunit is bound to the residual complex, i.e., to only one other subunit in the dimer versus three other subunits in the tetramer.

\section{Conclusions}

Using ESI it is possible to generate compact gas phase protein complexes carrying excess charge, usually in the form of protons. This charge accumulation is associated with a considerable energy density, which provides a driving force for the system to undergo change. Charge carrier ejection is a disfavored relaxation pathway due to the large enthalpic penalty associated with proton desolvation. Another possibility is the transition to a less compact structure (unfolding), or the ejection of a protein subunit. Upon collisional activation, it is common for protein complexes to follow relaxation pathways involving elements of the two aforementioned options, i.e., partial unfolding of a single subunit followed by subunit ejection $[30,33,37,44$, $49,51,53-55]$. The analogy of these processes to the breakup of charged solvent droplets has been noted [45].

The current work introduces a simple model that reproduces certain aspects associated with the fragmentation of gaseous protein complexes. By comparing experimental charge partitioning data with modeled proton distribution, it is possible to estimate the degree to which the ejected subunit has undergone unraveling at the scission point. Our findings imply that ejection occurs over a range of scission point structures that represent different degrees of unfolding. Data obtained for $\mathrm{Hb}$ suggest that these pre-dissociated states extend to conformations where the departing subunit is almost completely unraveled. Recent molecular dynamics work [75] as well as ion mobility measurements [51] provide further support for the existence of such highly expanded metastable structures.

An attractive feature of the framework developed here is that the most favorable charge configuration can be readily calculated using standard computational tools (Eq. 1). On the other hand, the level of structural detail in our model is very low. Features that have been omitted include the occurrence of unevenly spaced protonation sites, differences in the intrinsic gas phase basicities of individual residues, and thermal excitation effects that might induce some charge asymmetry even in the absence of unfolding. In the future, it will be interesting to extend electrostatic approaches of the type used here to more realistic protein descriptions [44, 46, 56], possibly even to full-scale molecular dynamics simulations [75]. It is hoped that those future endeavors will further advance the general understanding of gaseous biomolecular ions.

\section{Acknowledgements}

The authors acknowledge support for this work by the Natural Sciences and Engineering Research Council of Canada (NSERC), the Canada Foundation for Innovation (CFI), the Province of Ontario, the Canada Research Chairs Program, and The University of Western Ontario.

\section{References}

1. Kaddis, C.S., Loo, J.A.: Large Flying Proteins with ESI. Anal. Chem. 79, 1779-1784 (2007)

2. Heck, A.J.R.: Native mass spectrometry: a bridge between interactomics and structural biology. Nat. Methods 5, 927-933 (2008)

3. Benesch, J.L.P., Ruotolo, B.T., Simmons, D.A., Robinson, C.V.: Protein Complexes in the Gas Phase: Technology for Structural Genomics and Proteomics. Chem. Rev. 107, 3544-3567 (2007)

4. Fitzgerald, M.C., Chernushevich, I., Standing, K.G., Whitman, C.P., Kent, S.B.H.: Probing the Oligomeric Structure of an Enzyme by Electrospary Inonization Time-of-Flight Mass Spectrometry. Proc. Natl. Acad. Sci. U.S.A. 93, 6851-6856 (1996)

5. Ganem, B., Henion, J.D.: Going Gently into Flight: Analyzing Noncovalent Interactions by Mass Spectrometry. Bioorg. Med. Chem. 11, 311-314 (2003)

6. Katta, V., Chait, B.T.: Observation of the Heme-Globin Complex in Native Myoglobin by Electrospray-Ionization Mass Spectrometry. $J$. Am. Chem. Soc. 113, 8534-8535 (1991)

7. Light-Wahl, K.J., Schwartz, B.L., Smith, R.D.: Observation of the Noncovalent Quaternary Association of Proteins by Electrospray Ionization Mass Spectrometry. J. Am. Chem. Soc. 116, 5271-5278 (1994)

8. Fenn, J.B.: Electrospray Wings for Molecular Elephants (Nobel Lecture). Angew. Chem. Int. Ed. 42, 3871-3894 (2003)

9. Wilm, M., Mann, M.: Analytical Properties of the Nanoelectrospray Ion Source. Anal. Chem. 68, 1-8 (1996)

10. Daniel, J.M., Friess, S.D., Rajagopalan, S., Wendt, S., Zenobi, R.: Quantitative Determination of Noncovalent Binding Interactions Using Soft Ionization Mass Spectrometry. Int. J. Mass Spectrom. 216, 1-27 (2002)

11. Wang, W., Kitova, E.N., Klassen, J.S.: Influence of Solution and Gas Phase Processes on Protein-Carbohydrate Binding Affinities Determined by Nanoelectrospray Fourier Transform Ion Cyclotron Resonance Mass Spectrometry. Anal. Chem. 75, 4945-4955 (2003)

12. Jørgensen, T.J.D., Roepstorff, P., Heck, A.J.R.: Direct Determination of Solution Binding Constants for Noncovalent Complexes between Bacterial Cell Wall Peptide Analogues and Vancomycin Group Antibiotics by Electrospray Ionization Mass Spectrometry. Anal. Chem. 70, 4427-4432 (1998)

13. Liu, J., Konermann, L.: Protein-Protein Binding Affinities In Solution Determined by Electrospray Mass Spectrometry. J. Am. Soc. Mass Spectrom. 22, 408-417 (2011)

14. Frycak, P., Schug, K.A.: On-Line Dynamic Titration: Determination of Dissociation Constants for Noncovalent Complexes Using Gaussian Concentration Profiles by Electrospray Ionization Mass Spectrometry. Anal. Chem. 79, 5407-5413 (2007)

15. Chernushevich, I.V., Thomson, B.A.: Collisional Cooling of Large Ions in Electrospray Mass Spectrometry. Anal. Chem. 76, 1754-1760 (2004)

16. Tahallah, N., Pinkse, M., Maier, C.S., Heck, A.J.R.: The Effect of the Source Pressure on the Abundance of Ions of Noncovalent Protein Assemblies in an Electrospray Ionization Orthogonal Time-of-Flight Instrument. Rapid Commun. Mass Spectrom. 15, 596-601 (2001)

17. Collings, B.A., Douglas, D.J.: Conformation of Gas-Phase Myoglobin Ions. J. Am. Chem. Soc. 118, 4488-4489 (1996)

18. Bagal, D., Kitova, E.N., Liu, L., El-Hawiet, A., Schnier, P.D., Klassen, J.S.: Gas Phase Stabilization of Noncovalent Protein Complexes Formed by Electrospray Ionization. Anal. Chem. 81, 7801-7806 (2009)

19. Wang, W., Kitova, E.N., Klassen, J.S.: Determination of ProteinOligosaccharide Binding by Nanoelectrospray Fourier-Transform Ion Cyclotron Resonance Mass Spectrometry. Methods Enzymol. 362, 376$397(2003)$

20. Pan, J., Xu, K., Yang, X., Choy, W.Y., Konermann, L.: Solution-Phase Chelators for Suppressing Nonspecific Protein-Metal Interactions in Electrospray Mass Spectrometry. Anal. Chem. 81, 5008-5015 (2009) 
21. Sun, J., Kitova, E.N., Sun, N., Klassen, J.S.: Method for Identifying Nonspecific Protein-Protein Interactions in Nanoelectrospray Ionization Mass Spectrometry. Anal. Chem. 79, 8301-8311 (2007)

22. Ruotolo, B.T., Robinson, C.V.: Aspects of Native Proteins are Retained in Vacuum. Curr. Op. Chem. Biol. 10, 402-408 (2006)

23. Bernstein, S.L., Dupuis, N.F., Lazo, N.D., Wyttenbach, T., Condron, M.M., Bitan, G., Teplow, D.B., Shea, J.-E., Ruotolo, B.T., Robinson, C. V., Bowers, M.T.: Amyloid- $\beta$ Protein Oligomerization and the Importance of Tetramers and Dodecamers in the Aetiology of Alzheimer's Disease. Nat. Chem. 1, 326-331 (2009)

24. Ashcroft, A.E.: Mass Spectrometry and the Amyloid Problem-How Far Can We Go in the Gas Phase? J. Am. Soc. Mass Spectrom. 21, 1087-1096 (2010)

25. Rand, K.D., Pringle, S.D., Murphy, J.P., Fadgen, K.E., Brown, J., Engen, J.R.: Gas-Phase Hydrogen/Deuterium Exchange in a Traveling Wave Ion Guide for the Examination of Protein Conformations. Anal. Chem. 81, 10019-10028 (2009)

26. Talbot, F.O., Rullo, A., Yao, H., Jockusch, R.A.: Fluorescence Resonance Energy Transfer in Gaseous, Mass-Selected Polyproline Peptides. J. Am. Chem. Soc. 132, 16156-16164 (2010)

27. Sleno, L., Volmer, D.A.: Ion activation methods for tandem mass spectrometry. J. Mass Spectrom. 39, 1091-1112 (2004)

28. Xie, Y., Zhang, J., Yin, S., Loo, J.A.: Top-Down ESI-ECD-FT-ICR Mass Spectrometry Localizes Noncovalent Protein-Ligand Binding Sites. J. Am. Chem. Soc. 128, 14432-14433 (2006)

29. Wysocki, V.H., Jones, C.M., Galhena, A.S., Blackwell, A.E.: SurfaceInduced Dissociation Shows Potential to Be More Informative Than Collision-Induced Dissociation for Structural Studies of Large Systems. J. Am. Soc. Mass Spectrom. 19, 903-913 (2008)

30. Benesch, J.L.P.: Collisional Activation of Protein Complexes: Picking Up the Pieces. J. Am. Soc. Mass Spectrom. 20, 341-348 (2009)

31. Felitsyn, N., Kitova, E.N., Klassen, J.S.: Thermal Decomposition of a Gaseous Multiprotein Complex Studied by Blackbody Infrared Radiative Dissociation. Investigating the Origin of the Asymmetric Dissociation Behavior. Anal. Chem. 73, 4647-4661 (2001)

32. Sharon, M.: How Far Can We Go with Structural Mass Spectrometry of Protein Complexes? J. Am. Soc. Mass Spectrom. 21, 487-500 (2010)

33. Jurchen, J.C., Williams, E.R.: Origin of Asymmetric Charge Partitioning in the Dissociation of Gas-Phase Protein Homodimers. J. Am. Chem. Soc. 125, 2817-2826 (2003)

34. McLuckey, S.A., Goeringer, D.E.: Slow Heating Methods in Tandem Mass Spectrometry. J. Mass Spectrom. 32, 461-474 (1997)

35. Pagel, K., Hyung, S.-J., Ruotolo, B.T., Robinson, C.: Alternate Dissociation Pathways Identified in Charge-Reduced Protein Complex Ions. Anal. Chem. 82, 5363-5372 (2010)

36. Van den Heuvel, R.H.H., van Duijn, E., Mazon, H., Synowski, S.A., Lorenzen, K., Versluis, C., Brouns, S.J.J., Langridge, D., van der Oost, J., Hoyes, J., Heck, A.J.R.: Improving the Performance of a Quadrupole Time-of-Flight Instrument for Macromolecular Mass Spectrometry. Anal. Chem. 78, 7473-7483 (2006)

37. Jurchen, J.C., Garcia, D.E., Williams, E.R.: Further studies on the Origins of Asymmetric Charge Partitioning in Protein Homodimers. $J$. Am. Soc. Mass Spectrom. 15, 1408-1415 (2004)

38. Beardsley, R.L., Jones, C.M., Galhena, A.S., Wysocki, V.H.: Noncovalent Protein Tetramers and Pentamers with " $n$ " Charges Yield Monomers withn/4 and n/5 Charges. Anal. Chem. 81, 1347-1356 (2009)

39. Apostol, I.: Assessing the Relative Stabilities of Engineered Hemoglobins Using Electrospray Mass Spectrometry. Anal. Biochem. 272, 8-18 (1999)

40. Chernushevich, I.V., Loboda, A.V., Thomson, B.A.: An Introduction to Quadrupole Time-of-Flight Mass Spectrometry. J. Mass Spectrom. 36, 849-865 (2001)

41. Abzalimov, R.R., Frimpong, A.K., Kaltashov, I.A.: Gas-Phase Processes and Measurements of Macromolecular Properties in Solution: On the Possibility of False Positive and False Negative Signals of Protein Unfolding. Int. J. Mass Spectrom. 253, 207-216 (2006)

42. Gabelica, V., De Pauw, E.: Collision-Induced Dissociation of 16-mer DNA Duplexes with Various Sequences: Evidence for Conservation of the Double Helix Conformation in the Gas Phase. Int. J. Mass Spectrom. 219, 151-159 (2002)

43. Madsen, J.A., Brodbelt, J.S.: Asymmetric Charge Partitioning upon Dissociation of DNA Suplexes. J. Am. Soc. Mass Spectrom. 21, 11441150 (2010)
44. Sinelnikov, I., Kitova, E.N., Klassen, J.S.: Influence of Coulombic Repulsion on the Dissociation Pathways and Energetics of Multiproton Complexes in the Gas Phase. J. Am. Soc. Mass Spectrom. 18, 617-631 (2007)

45. Schwartz, B.L., Bruce, J.E., Anderson, G.A., Hofstadler, S.A., Rockwood, A.L., Smith, R.D., Chilkoti, A., Stayton, P.S.: Dissociation of Tetrameric Ions of Noncovalent Streptavidin Complexes Formed by Electrospray Ionization. J. Am. Soc. Mass Spectrom. 6, 459-465 (1995)

46. Wanasundara, S.N., Thachuk, M.: Free Energy Barrier Estimation for the Dissociation of Charged Protein Complexes in the Gas Phase. $J$. Phys. Chem. A 113, 3814-3821 (2009)

47. Boyd, R.K., Somogyi, Á.: The Mobile Proton Hypothesis in Fragmentation of Protonated Peptides: A Perspective. J. Am. Soc. Mass Spectrom. 21, 1275-1278 (2010)

48. Jørgensen, T.J.D., Gårdsvoll, H., Ploug, M., Roepstorff, P.: Intramolecular Migration of Amide Hydrogens in Protonated Peptides upon Collisional Activation. J. Am. Chem. Soc. 127, 2785-2793 (2005)

49. Jones, C.M., Beardsley, R.L., Galhena, A.S., Dagan, S., Cheng, G., Wysocki, V.H.: Symmetrical Gas-Phase Dissociation of Noncovalent Protein Complexes via Surface Collisions. J. Am. Chem. Soc. 128, 15044-15045 (2006)

50. Dodds, E.D., Blackwell, A.E., Jones, C.M., Holso, K.L., O'Brien, D.J., Cordes, M.H.J., Wysocki, V.H.: Determinants of Gas-Phase Disassembly Behavior in Homodimeric Protein Complexes with Related Yet Divergent Structures. Anal. Chem. 83, 3881-3889 (2011)

51. Ruotolo, B.T., Hyung, S.-J., Robinson, P.M., Giles, K., Bateman, R.H., Robinson, C.V.: Ion Mobility-Mass Spectrometry Reveals Long-Lived, Unfolded Intermediates in the Dissociation of Protein Complexes. Angew. Chem. Int. Ed. 46, 8001-8004 (2007)

52. Erba, E.B., Ruotolo, B.T., Barsky, D., Robinson, C.: Ion Mobility-Mass Spectrometry Reveals the Influence of Subunnit Packing and Charge on the Dissociation of Multiprotein complexes. Anal. Chem. 82, 97029710 (2010)

53. Ryce, S.A., Wyman, R.R.: Two Sphere Model for the Asymmetric Division of Electrically Charged Liquid Drops. Can. J. Phys. 48, 2571$2576(1970)$

54. Versluis, C., van der Staaji, A., Stokvis, E., Heck, A.J.R., de Craene, B.: Metastable Ion Formation and Disparate Charge Separation in the GasPhase Dissection of Protein Assemblies Studied by Orthogonal Timeof-Flight Mass Spectrometry. J. Am. Soc. Mass Spectrom. 12, 329-336 (2001)

55. Csiszar, S., Thachuk, M.: Using Ellipsoids to Model Charge Distributions in Gas Phase Protein Complex Ion Dissociation. Can. J. Chem. 82, 1736-1744 (2004)

56. Wanasundara, S.N., Thachuk, M.: Theoretical Investigations of the Dissociation of Charged Protein Complexes in the Gas Phase. J. Am. Soc. Mass Spectrom. 18, 2242-2253 (2007)

57. Konermann, L.: A Simple Model for the Disintegration of Highly Charged Solvent Droplets During Electrospray Ionization. J. Am. Soc. Mass Spectrom. 20, 496-506 (2009)

58. Kaltashov, I.A., Mohimen, A.: Estimates of Protein Surface Area in Solution by Electrospray Ionization Mass Spectrometry. Anal. Chem. 77, 5370-5379 (2005)

59. Voet, D. V., J. G., and Pratt, C.W. Fundamentals of Biochemistry, 2nd ed.; John Wiley and Sons, 2004

60. Gomez, A., Tang, K.: Charge and Fission of Droplets in Electrostatic Sprays. Phys. Fluids 6, 404-414 (1994)

61. Duft, D., Achtzehn, T., Muller, R., Huber, B.A., Leisner, T.: Coulomb Fission: Rayleigh Jets from Levitated Microdroplets. Nature 421, 128 (2003)

62. Li, D., Marquez, M., Xia, Y.: Capturing Electrified Nanodroplets under Rayleigh Instability by Coupling Electrospray with a Sol-Gel Reaction. Chem. Phys. Lett. 445, 271-275 (2007)

63. Gu, W., Heil, P.E., Choi, H., Kim, K.: Comprehensive Model for Fine Coulomb Fission of Liquid Droplets Charged to Rayleigh Limit. Appl. Phys. Lett. 91, 064104 (2007)

64. Kebarle, P., Verkerk, U.H.: Electrospray: From Ions in Solutions to Ions in the Gas Phase, What We Know Now. Mass Spectrom. Rev. 28, 898917 (2009)

65. Ahadi, E., Konermann, L.: Surface Charge of Electrosprayed Water Nanodroplets: A Molecular Dynamics Study. J. Am. Chem. Soc. 132, 11270-11277 (2010)

66. Antonini, E.; Brunori, M. Hemoglobin and Myoglobin in Their Reactions with Ligands; North-Holland Publishing Company: Amsterdam, London, 1971; Vol. 21 
67. Boys, B.L., Kuprowski, M.C., Konermann, L.: Symmetric Behavior of Hemoglobin $\alpha$ - and $\beta$-Subunits during Acid-Induced Denaturation Observed by Electrospray Mass Spectrometry. Biochemistry 46, 10675-10684 (2007)

68. Mark, K.J., Douglas, D.J.: Coulomb Effects in Binding of Heme in GasPhase Ions of Myoglobin. Rapid Commun. Mass Spectrom. 20, 111117 (2006)

69. Invernizzi, G., Samalikova, M., Brocca, S., Lotti, M., Molinari, H., Grandori, R.: Comparison of Bovine and Porcine $\beta$-Lactoglobulin: A Mass Spectrometric Analysis. J. Mass Spectrom. 41, 717-727 (2006)

70. Eaton, W.A., Henry, E.R., Hofrichter, J., Mozzarelli, A.: Is Cooperative Oxygen Binding by Hemoglobin Really Understood. Nat. Struct. Biol. 6, 351-358 (1999)
71. Perutz, M.F.: Stereochemistry of Cooperative Effects in Hemoglobin. Nature 228, 726-739 (1970)

72. Griffith, W.P., Kaltashov, I.A.: Highly Asymmetric Interactions Between Globin Chains During Hemoglobin Assembly Revealed by Electrospray Ionization Mass Spectrometry. Biochemistry 42, 1002410033 (2003)

73. Sakurai, K., Goto, Y.: Manipulating Monomer-Dimer Equilibrium of Bovine $\beta$-Lactoglobulin by Amino Acid Substitution. J. Biol. Chem. 277, 25735-25740 (2002)

74. Versluis, C., Heck, A.J.R.: Gas-phase dissociation of hemoglobin. Int. J. Mass Spectrom. 210/211, 637-649 (2001)

75. Wanasundara, S.N., Thachuk, M.: Toward an Improved Understanding of the Dissociation Mechanism of Gas Phase Protein Complexes. J. Phys. Chem. B 114, 11646-11653 (2010) 